\title{
Serum homocysteine, vitamin B12, folic acid levels and methylenetetrahydrofolate reductase (MTHFR) gene polymorphism in vitiligo
}

\author{
Ali Yasar ${ }^{\mathrm{a}}$, Kamer Gunduz ${ }^{\mathrm{a}, *}$, Ece Onur and ${ }^{\mathrm{b}}$ Mehmet Calkan $^{\mathrm{b}}$ \\ ${ }^{a}$ Department of Dermatology, Faculty of Medicine, Celal Bayar University, Manisa, Turkey \\ ${ }^{\mathrm{b}}$ Department of Biochemistry, Faculty of Medicine, Celal Bayar University, Manisa, Turkey
}

\begin{abstract}
The aim of this study was to determine serum vitamin B12, folic acid and homocysteine (Hcy) levels as well as MTHFR (C677, A1298C) gene polymorphisms in patients with vitiligo, and to compare the results with healthy controls. Forty patients with vitiligo and 40 age and sex matched healthy subjects were studied. Serum vitamin B12 and folate levels were determined by enzyme-linked immunosorbent assay. Plasma Hcy levels and MTHFR polymorphisms were determined by chemiluminescence and real time PCR methods, respectively. Mean serum vitamin B12 and Hcy levels were not significantly different while folic acid levels were significantly lower in the control group. There was no significant relationship between disease activity and vitamin B12, folic acid and homocystein levels. No significant difference in C677T gene polymorphism was detected. Heterozygote A1298C gene polymorphism in the patient group was statistically higher than the control group. There was no significant relationship between MTHFR gene polymorphisms and vitamin B12, folic acid and homocysteine levels. In conclusion, vitamin B12, folate and Hcy levels are not altered in vitiligo and MTHFR gene mutations (C677T and A1298C) do not seem to create susceptibility for vitiligo.
\end{abstract}

Keywords: Vitiligo, homocysteine, methylenetetrahydrofolate reductase, gene polymorphism, vitamin B12, folic acid

\section{Introduction}

Theories concerning autoimmune, cytotoxic, biochemical, neural, and oxidant-antioxidant mechanisms have been suggested in the etiopathogenesis of vitiligo. Roles of viruses and genetics have also been emphasized [7].

Folic acid and vitamin B12 levels have been reported to be lower in vitiligo patients when compared to the normal population [15], and also better results were achieved when folate and vitamin B12 are incorporated with vitiligo treatment [8]. Folate and vitamin B12 deficiencies lead increase in plasma homocysteine (Hcy)

* Corresponding author: Prof. Kamer Gündüz, Uncubozköy Mah. 5501 Sok, No: 29/5 Manisa-Turkey. Tel.: +90 236 2341012; Fax: +90 236 2338040; E-mail: kamergunduz@gmail.com. levels. Increase of Hcy causes activation of various cytokines and lipid peroxidation and increased reactive oxygen radicals may have toxic effects on melanocytes. Another likely mechanism is inhibition of thyrosinase by Hcy [16].

Methylenetetrahydrofolate reductase (MTHFR) is an enzyme affecting Hcy and methionine metabolisms. It catalyzes reduction of 5,10-methylene THF to 5-methyl THF. 5-methyl THF acts as a methyl donor during synthesis of methionine from Hcy. In case of genetic polymorphism, MTHFR enzyme activity decreases and failure to synthesize methionine from Hcy causes Hcy levels to increase [12]. Folic acid, vitamin B12 and vitamin B6 deficiencies and reduced enzyme activities decrease catabolism of Hcy and cause an increase in the intracellular Hcy concentration [17].

After MTHFR gene was projected to be located in the 36.3 region on the short arm of chromosome 1 , it 
has been shown that C677T polymorphism occurs as a result of substitution of cytosine (C) with thymine (T) on nucleotide 677 of this gene. In homozygote (TT) individuals with C677T polymorphism, total plasma Hcy levels were found significantly higher whereas Hcy levels were similar in heterozygotes (CT) and non-mutated (CC). In recent years, A1298C polymorphism was identified as the second most frequent mutation seen on the same gene. In this new mutation type, cytosine (C) substitutes adenine (A) on nucleotide 1298. Similar to C677T mutation, MTHFR activity decreases more significantly in homozygote (CC) individuals compared to heterozygote (AC) and regular (AA) ones [5].

In vitiligo, low levels of vitamin B12 and folate might decrease the synthesis of methionine from Hcy, and Hcy levels would increase accordingly. It was suggested that Hcy suppresses histidase and thyrosinase activity in the skin and hinder melanin synthesis [16].

The aim of this study was to determine serum vitamin B12, folic acid and Hcy levels as well as MTHFR (C677, A1298C) gene polymorphisms in patients with vitiligo, and to understand their possible roles in the disease etiology and to compare the results with the healthy controls.

\section{Methods}

The local ethics committee approved the study design and all study participants signed an informed consent form. Forty vitiligo patients and age-sex matched 40 healthy volunteers were included in the study.

Patients with diseases increasing Hcy levels such as genetic amino acid metabolism disorders, hypertension, diabetes, cardiovascular disease, kidney failure, deep vein thrombosis, Behçet disease, and psoriasis were not included in the study.

Age, gender, onset age and family history of vitiligo were recorded. Disease activity was assessed as follows: Cases with new lesions or growth in the existing lesions within the last 3 months were considered as active vitiligo whereas cases with reduced lesions were classified as regressive vitiligo and cases with no changes were considered as stable vitiligo. According to clinical distribution, the patients were classified as focal, segmental, acrofacial and generalized.

Venous blood samples were drawn into plain and EDTA containing tubes following $12 \mathrm{~h}$ of fasting. Specimens were centrifuged at 4,000 rpm for 10 minutes within 2 hours after sampling. The sera and plasma were frozen at $-80^{\circ} \mathrm{C}$ until analysis.

Serum vitamin B12 and folate levels were determined by enzyme-linked immunosorbent assay using a Unicell DXI 800 (Beckman Coulter) autoanalyzer. Plasma Hcy levels were determined by chemiluminescence method, using automatic immunoassay analyzer (Immulite-2000, Diagnostic Products Corporation LA, USA).

\subsection{MTHFR polymorphism analysis}

Genomic DNA was isolated (extracted) from whole blood samples placed in EDTA containing tubes by using High Pure PCR Template Preparation Kit (Cat. No: 11796828001, Roche Diagnostics). They were kept at $-80^{\circ} \mathrm{C}$ until analyzed. MTHFR polymorphisms were assessed by real time PCR method using LightCycler 1.5 analyzer (Roche Diagnostics). PCR primers and hybridization probes were synthesized by Tib Molbiol (Berlin, Germany). MTFHR C677T and MTHFR A1298C Kits (Cat. No: 40-0096-16 ve 40-0269-16) were used in combination with LightCycler Faststart DNA Master Hybridization Probes kit (Roche Diagnostics).

\subsection{Statistical analysis}

SPSS 16.0 software was used for statistical evaluation. Data analysis was carried by using Student t-test, Kruskal-Wallis test, Mann-Whitney U test, chi-square test and Oneway-Anova test. P values smaller than 0.05 were considered as statistically significant.

\section{Results}

The mean age of the vitiligo patients ( 23 female, 17 male) was $27.77 \pm 13.44$ (range 10-56). In the control group ( 22 female, 18 male), the mean age was $25.42 \pm 4.48$ (range 20-41). Vitiligo cases and the control group were found to be similar in respect to age and gender $(p>0.05)$.

Onset age of vitiligo ranged between 2 and $56(19.60$ $\pm 13.39)$. Family history was positive in 7 (17.5\%) patients. None of the male patients had positive family history. This difference between male and female patients was found to be significant $(p=0.012)$.

According to the clinical distribution, 4 (10\%) patients were focal type, $1(2.5 \%)$ was segmental type, 8 (20\%) were acrofacial type and $27(67.5 \%)$ were gen- 
Table 1

Vitamin B12, folic acid and homocysteine levels (mean \pm SD) for the patient and the control groups

\begin{tabular}{lcc}
\hline & Patient $(n=40)$ & Control $(n=40)$ \\
\hline Vitamin B 12 & $212.90 \pm 81.67$ & $241.15 \pm 126.23$ \\
Folate & $6.59 \pm 2.78$ & $5.39 \pm 2.41$ \\
Homocysteine & $9.35 \pm 5.70$ & $10.96 \pm 5.31$ \\
\hline
\end{tabular}

Table 2

C677T and A1298C gene polymorphisms in the patient and the control groups

\begin{tabular}{llcc}
\hline & & Patient $\mathrm{n}(\%)$ & Control $\mathrm{n}(\%)$ \\
\hline C677T polymorphism & CC & $25(62.5)$ & $20(50)$ \\
& CT & $13(32.5)$ & $15(37.5)$ \\
& TT & $2(5)$ & $5(12.5)$ \\
A1298C gene polymorphism & AA & $10(25)$ & $18(45)$ \\
& AC* & $25(62.5)$ & $11(27.5)$ \\
& CC & $5(12.5)$ & $11(27.5)$ \\
\hline
\end{tabular}

${ }^{*} P=0.007$ (Chi-square test).

eralized type vitiligo. Five (12.5\%) patients were assessed as active, $6(15 \%)$ patients as regressive and 29 $(72.5 \%)$ as stable according to disease activity. Vitamin B12, folic acid and Hcy levels in the patient and the control groups are shown in Table 1. C677T and A1298C gene polymorphisms are shown in Table 2. In respect to A1289C polymorphism, heterozygote (AC) ratio in the patient group was found to be significantly higher $(p=0.007)$.

No statistically significant relation was found between the gene polymorphisms and vitamin B12, folic acid and Hcy levels (Table 3).

\section{Discussion}

Although many theories have been suggested, the etiopathogenesis of vitiligo is not fully understood yet. An association has been suggested between vitiligo and pernicious anaemia and/or folic acid deficiency [13, 15]. Montes et al. [13] reported lower vitamin B12, folate and ascorbic acid levels in the vitiligo patients and achieved significant repigmentation in half of the patients as a result of oral folic acid and ascorbic acid treatment together with vitamin B12 therapy. Juhlin et al. [8] stated that significant repigmentation was achieved in 52 of 100 vitiligo patients, and spread of the disease was stopped in 64 patients with vitamin B12 and folic acid supplementation together with sunbathing. However, this issue remains controversial. There are studies that show no differences in vitamin B12 and folate levels between vitiligo and control groups $[1,6$, $10]$.
In the present study, although mean vitamin B12 level was lower than the control group, the difference was not statistically significant. Interestingly, folic acid levels were significantly lower in the control group. There was no significant relation between the disease activity and vitamin B12 and folate levels. We found folic acid levels significantly lower in the male patients like Kim et al. [10].

Vitamin B12 and folic acid are major determinants of homocysteine levels, and a nutritional deficiency in either of these two vitamins results in hyperhomocysteinaemia [9]. Shaker and El-Tahlawi [16] reported significantly higher Hcy levels in patients with especially progressive vitiligo. They recommended routine determination of Hcy levels in patients with vitiligo and addition of Hcy lowering agents such as vitamin B6, B12 and folic acid to the vitiligo treatment protocol. In a recent study, Karadag et al. [9] reported significantly higher homocysteine but lower vitamin B12 and holotranscobalamine levels in vitiligo and suggested that there might be a common pathogenetic susceptibility to both vitiligo and B12 deficiency.

On the other hand, Balci et al. [1] found no significant differences in the Hcy, folic acid, and vitamin B12 levels between the vitiligo patients and the controls. The authors suggested that ethnic differences might have effects on Hcy levels. Like Balci et al., we found no significant differences in Hcy levels between the vitiligo patients and the control group. In the present study, Hcy levels were significantly higher in males both in the patient and the control groups.

MTHFR gene polymorphisms were shown to reduce the enzyme activity and increase total Hcy levels. Castro et al. [3] investigated C667T and A1298C MTHFR gene polymorphisms in healthy volunteers and reported higher homocysteine levels in mutant homozygotes (TT, CC) and significantly lower plasma folate levels in mutant homozygotes (TT). However, in the study of Chango [4], although homocysteine levels in 677TT and 1298CC genotype subjects were higher, no significant differences were observed among different genotypes.

Among dermatological diseases, the relation between MTHFR gene polymorphisms and Hcy levels was investigated in patients with Behçet Disease (BD). Koubaa et al. [11] reported that homozygosity for the $\mathrm{T}$ allele of the MTHFR gene was more frequent in BD patients and it was associated with significantly higher Hcy levels. Ozkul et al. [14] stated that in BD patients with ocular involvement, the frequencies of MTHFR TT homogenetic type were significantly higher than 
Table 3

Relation between the gene polymorphisms and vitamin B12, folic acid and Hcy levels

\begin{tabular}{lcrccr}
\hline & Genotype & $\mathrm{n}$ & Vitamin B12 & Folic acid & Homocysteine \\
\hline C667T & CC & 25 & $223,32 \pm 89,43$ & $6,85 \pm 2,68$ & $7,97 \pm 2,98$ \\
& CT & 13 & $188,85 \pm 67,00$ & $6,27 \pm 3,19$ & $12,54 \pm 8,39$ \\
& TT & 2 & $239,00 \pm 52,32$ & $5,53 \pm 1,43$ & $5,93 \pm 2,41$ \\
& Total & 40 & $212,90 \pm 81,67$ & $6,59 \pm 2,78$ & $9,35 \pm 5,70$ \\
A1298C & AA & 10 & $258,90 \pm 89,35$ & $6,63 \pm 2,31$ & $9,10 \pm 5,41$ \\
& AC & 25 & $186,80 \pm 65,41$ & $6,43 \pm 2,86$ & $9,60 \pm 6,24$ \\
& CC & 5 & $251,40 \pm 100,02$ & $7,36 \pm 3,63$ & $8,66 \pm 4,03$ \\
& Total & 40 & $212,90 \pm 81,67$ & $6,59 \pm 2,78$ & $9,35 \pm 5,70$ \\
\hline
\end{tabular}

Values are expressed as mean $\pm \mathrm{SD}$. Kruskal-Wallis test.

C677T Polymorphism $P=0,724$ (Vitamin B12), $P=0,755$ (Folate), $P=0,127$ (Homocysteine);

A1298C Polymorphism $P=0,065$ (Vitamin B12), $P=0,800$ (Folate), $P=0,987$ (Homocysteine).

those in BD patients without ocular involvement or the controls and concluded that elevated serum levels of Hcy seemed to be a result of C677T polymorphism of the MTHFR gene. On the other hand, in a recent study, no differences were found between BD patients and control subjects concerning the MTHFR gene polymorphisms [2].

To our knowledge, this is the first study investigating the MTHFR gene polymorphism in vitiligo. Regarding the C677T polymorphism, no statistically significant difference was found between the patient and the control groups. Although not statistically significant, TT genotype of C677T was higher in the control group than the vitiligo patients $(12.5 \%$ vs. $5 \%)$. This finding might explain the lower folate levels in the control group. Heterozygote (AC) genotype rate for A1298C polymorphism was significantly higher in vitiligo patients than the control group, but Hcy levels did not increase in parallel with this difference. There was no significant relation between the C677T and A1298C polymorphisms and plasma Hcy levels.

In conclusion, we suggest that vitamin B12, folate and Hcy levels are not altered in vitiligo and MTHFR gene mutations (C677T and A1298C) do not create susceptibility for vitiligo.

\section{References}

[1] D.D. Balci, Z. Yonden, J.Z. Yenin, N. Okumus, Serum homocysteine, folic acid and vitamin B12 levels in vitiligo, Eur J Dermatol 19 (2009), 382-3.

[2] P. Caramaschi, G. Poli, A. Bonora, A. Volpe, I. Tinazzi, S. Pieropan, L.M. Bambara, D. Biasi, A study on thrombophilic factors in Italian Behcet's patients, Joint Bone Spine 77 (2010), 330-4.

[3] R. Castro, I. Rivera, P. Ravasco, C. Jakobs, H.J. Blom, M.E. Camilo, I.T. de Almeida, 5,10-Methylenetetrahydrofolate reductase $677 \mathrm{C} \rightarrow \mathrm{T}$ and $1298 \mathrm{~A} \rightarrow \mathrm{C}$ mutations are genetic determinants of elevated homocysteine, QJM 96 (2003), 297-303.
[4] A. Chango, F. Boisson, F. Barbé, D. Quilliot, S. Droesch, M. Pfister, N. Fillon-Emery, D. Lambert, S. Frémont, D.S. Rosenblatt, J.P. Nicolas, The effect of $677 \mathrm{C} \rightarrow \mathrm{T}$ and $1298 \mathrm{~A} \rightarrow \mathrm{C}$ mutations on plasma homocysteine and 5,10methylenetetrahydrofolate reductase activity in healthy subjects, Br J Nutr 83 (2000), 593-6.

[5] G. Friedman, N. Goldschmidt, Y. Friedlander, A. Ben-Yehuda, J. Selhub, S. Babaey, M. Mendel, M. Kidron, H. Bar-On, A common mutation A1298C in human methylenetetrahydrofolate reductase gene: association with plasma total homocysteine and folate concentrations, J Nutr 129 (1999), 1656-61.

[6] M. Gonul, S.K. Cakmak, S. Soylu, A. Kilic, U. Gul, Serum vitamin B12, folate, ferritin and iron levels in Turkish patients with vitiligo, Indian J Dermatol Venereol Leprol 76 (2010), 448.

[7] R.M. Halder, S.J. Taliaferro, Vitiligo, in: Fitzpatrick's Dermatology in General Medicine, K. Wolff, L.A. Goldsmith, S.I. Katz, B.A. Gilchrest, A.S. Paller, D.J. Leffell, eds, McGraw Hill, New York, 2008, pp. 616-22.

[8] L. Juhlin and M.J. Olsson, Improvement of vitiligo after oral treatment with vitamin B12 and folic acid and the importance of sun exposure, Acta Derm Venereol 77 (1997), 460-2.

[9] A.S. Karadag, E. Tutal, D.T. Ertugrul, K.O. Akin, S.G. Bilgili, Serum holotranscobalamine, vitamin B12, folic acid and homocysteine levels in patients with vitiligo, Clin Exp Dermatol 37 (2011), 62-64.

[10] S.M. Kim, Y.K. Kim, S.K. Hann, Serum levels of folic acid and vitamin B12 in Korean patients with vitiligo, Yonsei Med J 40 (1999), 195-8.

[11] N. Koubaa, S. Hammami, A. Nakbi, K. Ben Hamda, S. Mahjoub, T. Kosaka, M. Hammami, Relationship between thiolactonase activity and hyperhomocysteinemia according to MTHFR gene polymorphism in Tunisian Behçet's disease patients, Clin Chem Lab Med 46 (2008), 187-92.

[12] U.K. Misra, J. Kalita, A.K. Srivastava, S. Agarwal, MTHFR gene polymorphism and its relationship with plasma homocysteine and folate in a North Indian population, Biochem Genet 48 (2010), 229-35.

[13] L.F. Montes, M.L. Diaz, J. Lajous, N.J. Garcia, Folic acid and vitamin B12 in vitiligo: A nutritional approach, Cutis 50 (1992), 39-42.

[14] Y. Ozkul, C. Evereklioglu, M. Borlu, S. Taheri, M. Calis, M. Dündar, O. Ilhan, 5,10- Methylenetetrahydrofolate reductase C677T gene polymorphism in Behçet's patients, Br J Ophthalmol 89 (2005), 1634-7.

[15] H.H. Park and M.H. Lee, Serum levels of vitamin B12 and folate in Korean patients with vitiligo, Acta Derm Venereol 85 (2005), 66-7. 
[16] O.G. Shaker and S.M. El-Tahlawi, Is there a relationship between homocysteine and vitiligo? A pilot study, Br J Dermatol 159 (2008), 720-4.

[17] O. Stanger, W. Herrmann, K. Pietrzik, B. Fowler, J. Geisel,
J. Dierkes, M. Weger, Clinical use and rational management of homocysteine, folic acid, and B vitamins in cardiovascular and thrombotic diseases, Z Kardiol 93 (2004), 439-53. 


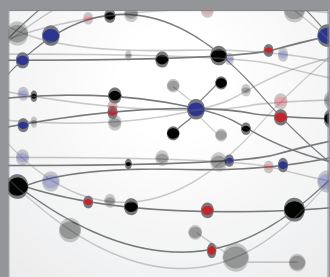

The Scientific World Journal
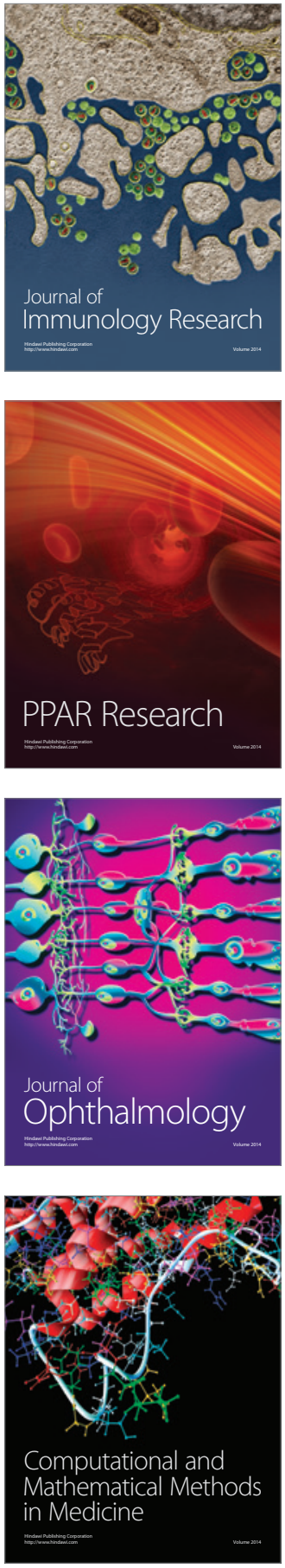

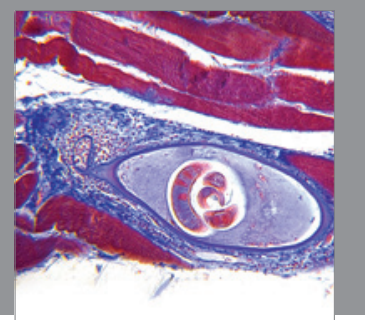

Gastroenterology

Research and Practice
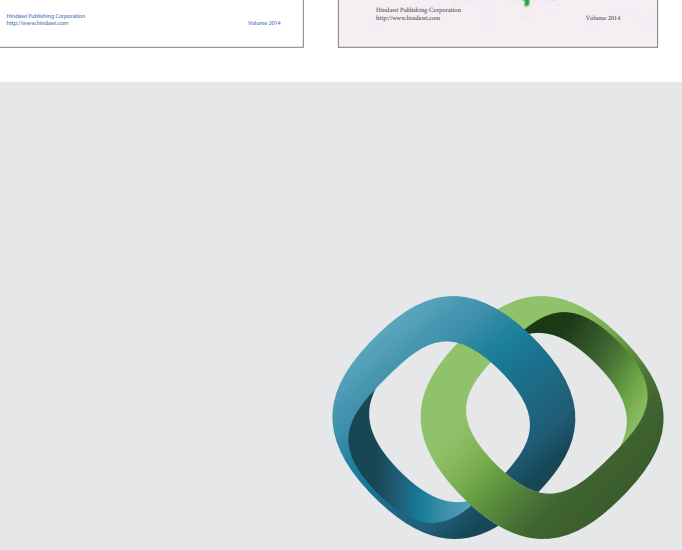

\section{Hindawi}

Submit your manuscripts at

http://www.hindawi.com
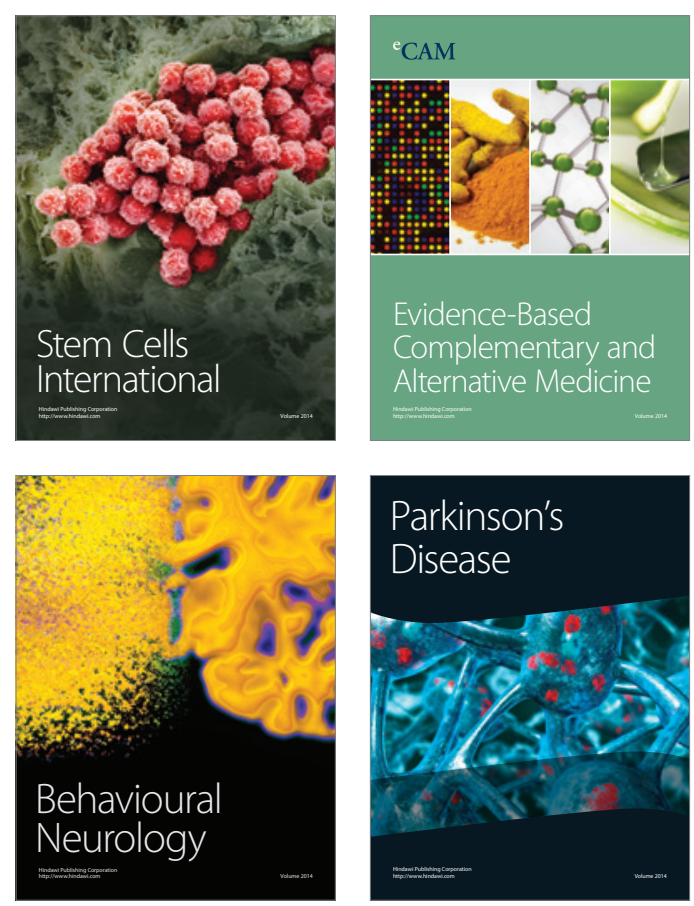

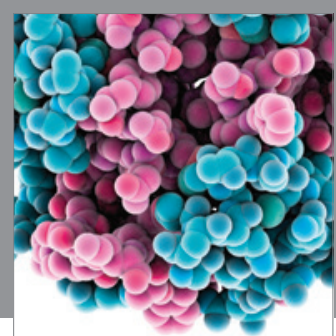

Journal of
Diabetes Research

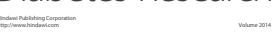

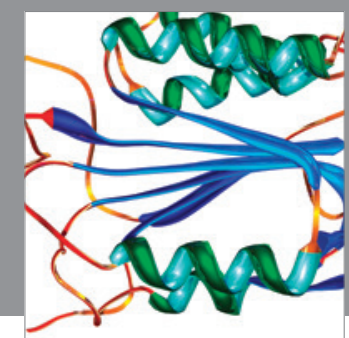

Disease Markers
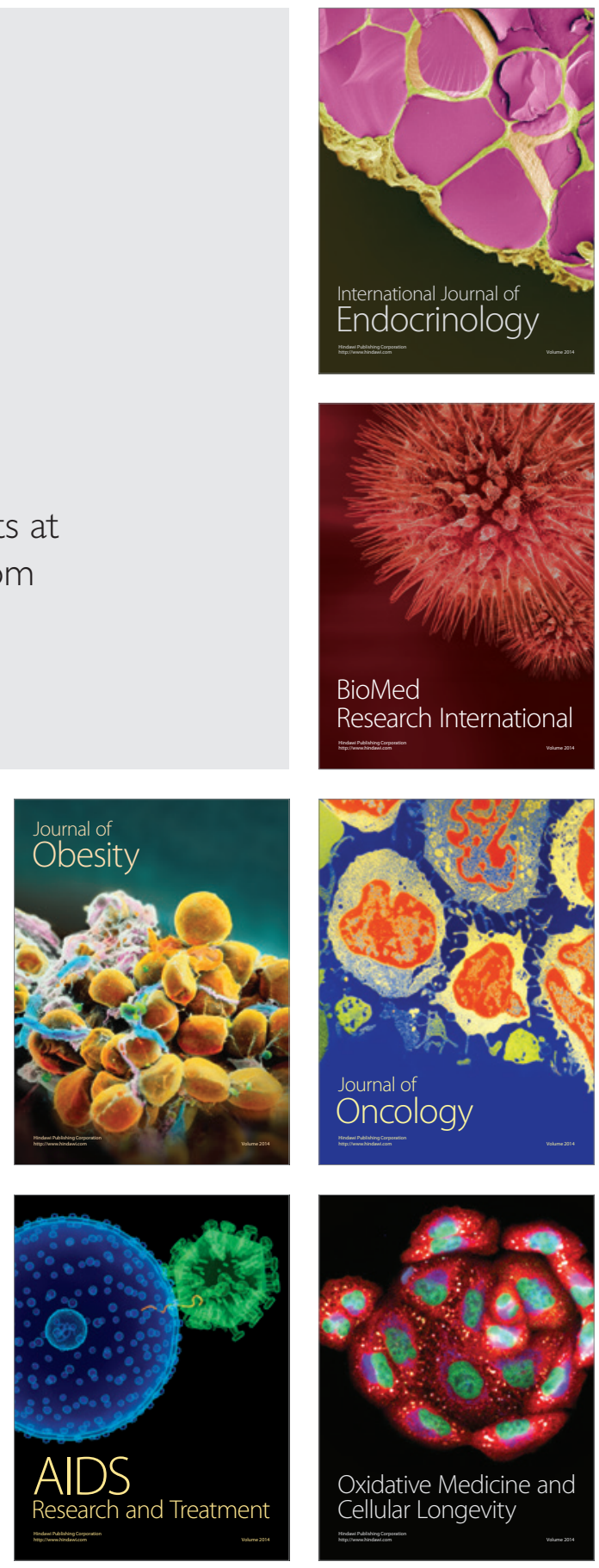